\title{
Proximal Loop Ileostomy-A Life Saving Approach in ComplicatedEnterocutaneous Fistulas
}

\author{
Dr Sudershan Kapoor \\ Associate Professor, Department of Surgery, Govt. Medical CollegeAmritsar,India
}

\begin{abstract}
An enterocutaneous fistula (ECF) is a potentially catastrophic postoperative complication. Although the morbidity and mortality associated with ECF have decreased over the past 50 years with modern medical and surgical care, the overall mortality is still surprisingly high, up to $39 \%$ in recent literature. It seems prudent, then, for every surgeon to have a thorough grasp of optimal treatment strategies for ECF to minimize their patients' mortality. Ultimately, the algorithm must begin with prevention. Once an ECF is diagnosed, the key to managemnet is to resuscitate and treat sepsis, to control fistula output, to optimize the patient medically and nutritionally and definitive restoration of gastrointestinal continuity. Special mention is given in this article to complicated fistulas such as those with high output,abdominal sepsis and large abdominal defects. There is stress on diversion of fecal matter through ileostomy at a normal site of intestines at a distance of fistula site and damage/devitalized and inflammed gut(Proximal loop ileostomy) and fistula repair at comparatively early stage to prevent the further complications of sepsis and malnutrition. This plan gives a framework for the difficult task of successfully treating the postoperative ECF with a multidisciplinary approach.

Keywords: Enterocutaneous fistula, nutritional support, sepsis, Proximal loop ileostomy
\end{abstract}

\section{Introduction}

Enterocutaneous Fistulas are a common presentation in general surgical wards, and despite advances in the management of these lesions, they are still responsible for a significant mortality rate, ranging from $5-20 \%$, due to associated sepsis, nutritional abnormalities, and electrolyte imbalances.Entero-cutaneous fistulas are more commonly seen in post-operative setting.Morbidity associated with fistulas is significant.; the principle cause of death are sepsis and malnutrition(9).

Particular difficult are complicated fistulas of those associated with large abdominal defects.Mortality rates in these cases may reach $60 \%-80 \%$. The current treatment of intestinal fistulas coupled with a multidisciplinary approach has helped to decrease the mortality rate to $15-30 \%$.

Earlier study suggests that about $95 \%$ of ECFs were postoperative and ileum was found to be the most common site of ECF. ${ }^{(1)}$ Forty-nine percent of fistulas were high output and $51 \%$ were low output.

Enterocutaneous fistulas (ECFs) can occur as a complication following any type of surgery on the GI tract. Indeed, more than $75 \%$ of all ECFs arise as a postoperative complication, while about $15-25 \%$ of them result from abdominal trauma or occur spontaneously in relation to cancer, irradiation, inflammatory bowel disease, or ischemic or infective conditions. The etiology of ECFs can thus be characterized as postoperative, traumatic, or spontaneous.(3)

\section{Postoperative causes}

Postoperative causes of ECFs include the following:

- $\quad$ Disruption of anastomosis

- Inadvertent enterotomy - Especially occurs in patients with adhesions, when dissection can cause multiple serosal tears and an occasional full-thickness tear

- Inadvertent small bowel injury - Occurs during abdominal closure, especially after ventral hernia repair malnutrition.

Enteroatmospheric fistula (EAF), a special subset of ECF, is defined as a communication between the gastrointestinal (GI) tract and the atmosphere. It can occur as a complication of "damage control" laparotomy (DCL) and results in significant morbidity and mortality.(5)

Their etiology is complex and ranges from persistent abdominal infection, anastomotic dehiscence, and adhesions of the bowel to fascia with a laparostoma. As EAFs almost never close spontaneously, definitive repair usually requires major surgical intervention.(8)

The type of ECF, as based on the output of the enteric contents, also determines the patient's health status and how the patient may respond to therapy. ECFs are usually classified into 3 categories, as follows ${ }^{[2]}$ :

- Low-output fistula $(<200 \mathrm{~mL} /$ day $)$,

- $\quad$ Moderate-output fistula (200-500mL/day)

- $\quad$ High-output fistula $(>500 \mathrm{~mL} /$ day $)$ 
A high-output fistula increases the possibility of fluid and electrolyte imbalance and Etiology(7)

Type I ECF originate from esophageal, gastric and duodenal sources;

Type II from small bowel;

Type III from large bowel; and

Type IV from large abdominal wall defects greater than $20 \mathrm{~cm}$

\section{ComplicatedEnterocutaneousfistulas:}

- Complete distal intestinal obstruction.

- $\quad$ Adjacent infection or abscess.

- $\quad$ Fistulas associated with large abdominal wall defects.

- Fistulas associated with the loss of continuity of the gut

- Fistulas associated with the gut pathology.

- $\quad$ Fistular opening more than $2 \mathrm{~cm}$.

- Continued high output from the fistula in spite of nothing orally and on continuous parenteral nutrition.

- Bleeding from the fistula.

- Epethialised tracts.

- Adjacent foreign material like mesh or sutures.

\section{Role of Surgery}

Proximal diversion of fecal matter by making ostomies can reduce the complications related to continuous exposure to sepsis in complex fistulas.

Early surgical intervention is required in all complicated enterocutaneousfistulas.Nonsurgical therapy may allow for spontaneous closure of the fistulas,though this can be expected in less than $30 \%$ cases and that also depends upon the nutritional status of the patient,sepsis and underlying cause.Drainage of intraperitoneal abscesses, resection of devitalized parts, repair of the damaged parts of gut can be attempted at the same sitting along the proximal diversion. This avoids the subsequent laparotomies at a later stage.Closure of the ostomy can be done after 8-12 weeks when the general condition of the patient improves(never before 6 weeks) and healing of the distal segment of gut.After doing proximal ostomy, enteral nutrition can be started at the earliest.Early start of enteral nutrition decreases the bacterial translocation and the tropic effects on the intestinal mucosa.

\section{Aims And Objectives:}

A prospective study of the role of early proximal loop ileostomies in cases of complicated entero-cutaneous fistulas-A study of 60 cases (2005-2012).

\section{Material And Methods:}

This study was conducted on 60 cases of complicatedenterocutaneous fistulas ,all were psot -operative and majority were of high output type.After resuscitation and supportive management,early exploration was done.Resection of devitalisedpart,drainage of infected foci,repair of the damaged part of intestine and proximal loop ileostomy was done.Routine and other relevant investigations were done.

\section{Preparation Of The Patient:}

Surgical planning for fistula repair was individualised according to the pt's condition and intraoperative findings.(6)

Appropriate pre-operative assessment included(10):

1.Ensure that the patient is euvolemic.

2. Blood transfusions and TPN as required.

3.Proper coverage of antibiotics.

4. Central venous line

5. Foley's catheterization

\section{Intra-Operative Findings:}

During exploration, following points were noted:

1.Collection in peritoneal cavity.

2.Site, size and number of fistulas.

3.Condition of gut.

4.Intra peritoneal and interloop abscesses.

5.Any evidence of obstruction distal to fistula

6. Condition of other viscera 


\section{Procedure done:}

Drainage of collections.

Exploration,lysis of adhesions and mobilisation of the entire gut.

Resection of devitalised part. sutures.

Repair of the fistular segments or resection anastamosis in single layer with interrupted non absorbable

Proximal loop ileostomy (PLI) from the comparatively healthy part of gut( at least30 $-40 \mathrm{~cm}$ from the damaged/inflamed/diseased gut).

Abdominal drain in pelvis.

Closure of the main wound in two layers

\section{Bservations:}

- AGE INCIDENCE: 10-50 yrs (Majority 20-40 yrs).

- $\quad$ SEX INCIDENCE: Males:47 Females:13

- SEX INCIDENCE: Males:47 Females:13

- $\quad$ FISTULA OUTPUT: High output:50 cases

- Low output:10 cases

- ETIOLOGY : Post-operative
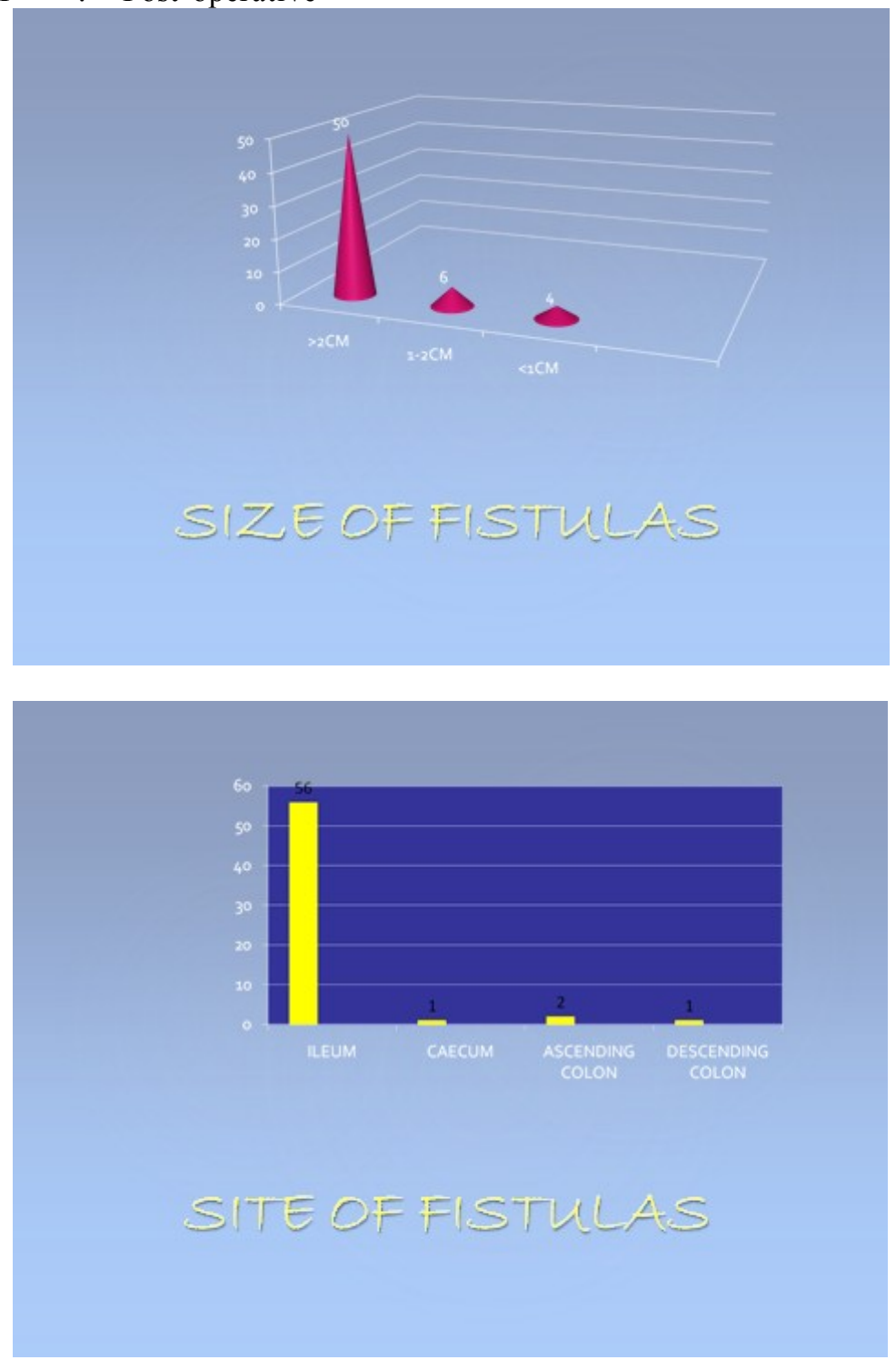
CONSERVATIVE

PRIMARY REPAIR

ILEOSTOMES

SURGICAL

PROCEDURES

- RE-EXPI_ORATION AFTER PRIMARY REPAIR -6 cases

- ILEOSTOMY CLOSURE (After 8-12 wks) :50 cases

- POST-OPERATIVE WOUND INFECTION 23 cases

- POST-OPERTAIVE WOUND DEHISCENCE:

COMPLETE $: 1$ Case

PARTIAL : 14 Cases

- FOLLOW UP (ANY FECAL DISCHARge AFTER ILEOSTOMY CLOSURE):

Only 1 case having small amount of feculent discharge with the normal bowel habits of patient- managed conservatively.

- PERI-OPERATIVE MORTALITY:

6 cases. (due to septicaemia)

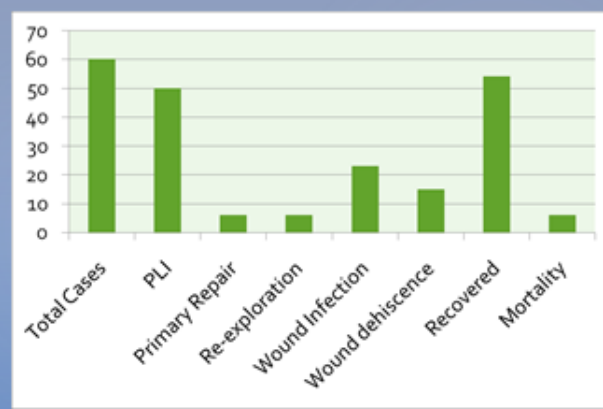




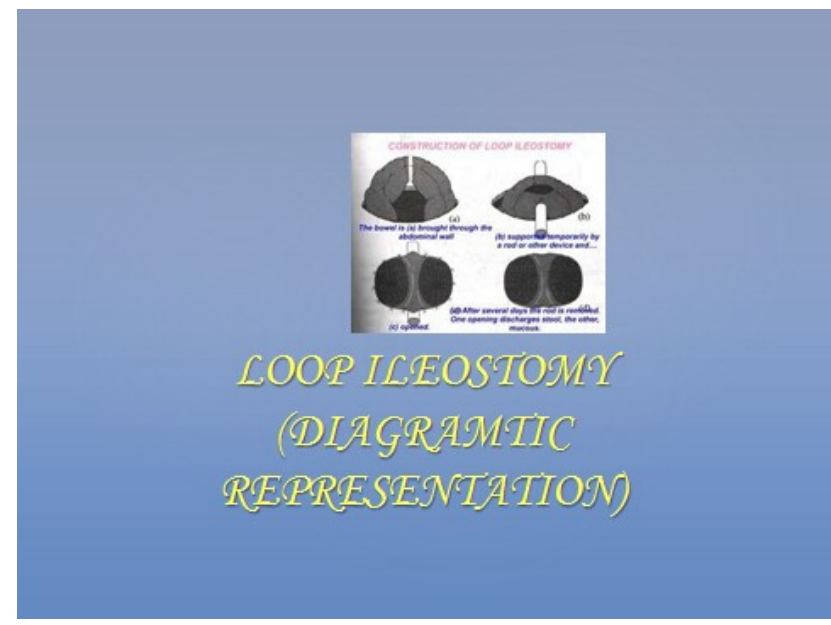

\section{Discussion}

- In our study, majority of the patients presented with complicated type of entero-cutaneous fistulas.

- In 50 out of 60 cases, exploration with drainage of intra-peritoneal collections, resection of the devitalized part and repair of the gut was done and proximal loop ileostomies were created.

- These patients did well post operatively without any major post-operative complications in most of the cases.

- Those 6 cases in whom primary repair was done, had to be re-explored and treated on the same line of proximal loop ileostomy. They also responded well post-operatively except 6 cases( died during the course of treatment.)

- Proximal loop ileostomy (P L I) with repair of the damaged part of gut showed good results and less morbidity and mortality in the present study.

- P L I also avoids re-laparotomies at later stages as ileostomy closure can be carried out by exploring the same area of ileostomy site.

- P L I is better than exteriorization of the fistula segments as that part of gut is badly inflamed adherent and has septic focus, so more chances of ileostomy related complications.

- P L I provides sufficient time for the healing of repaired part of gut and inflammation of adjacent loops of gut to settle down.

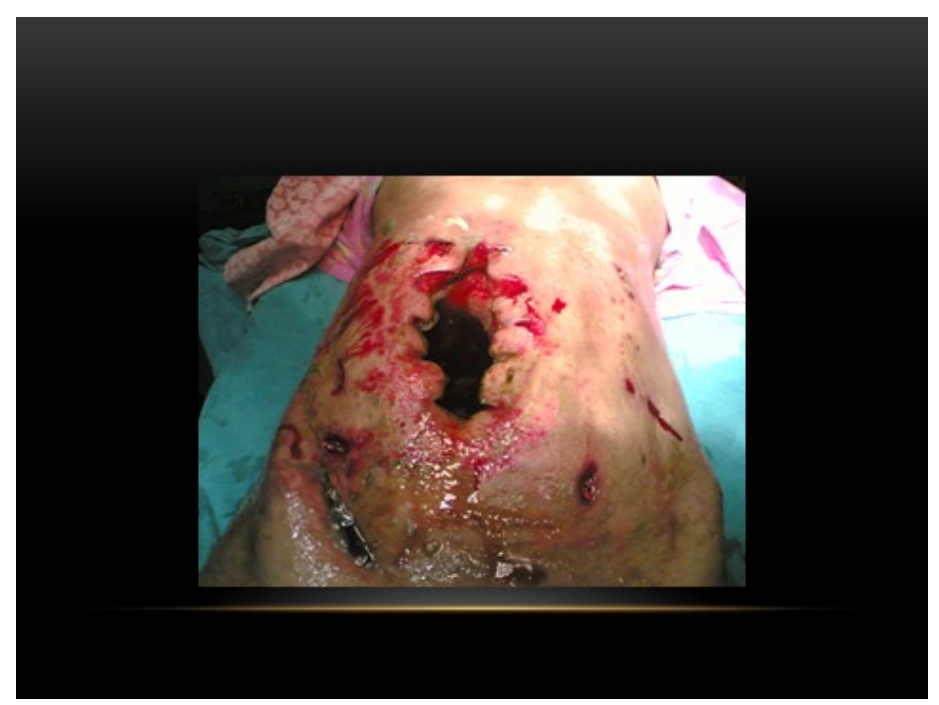

Fig 1..Enterocutaneous Fistula Case-1 


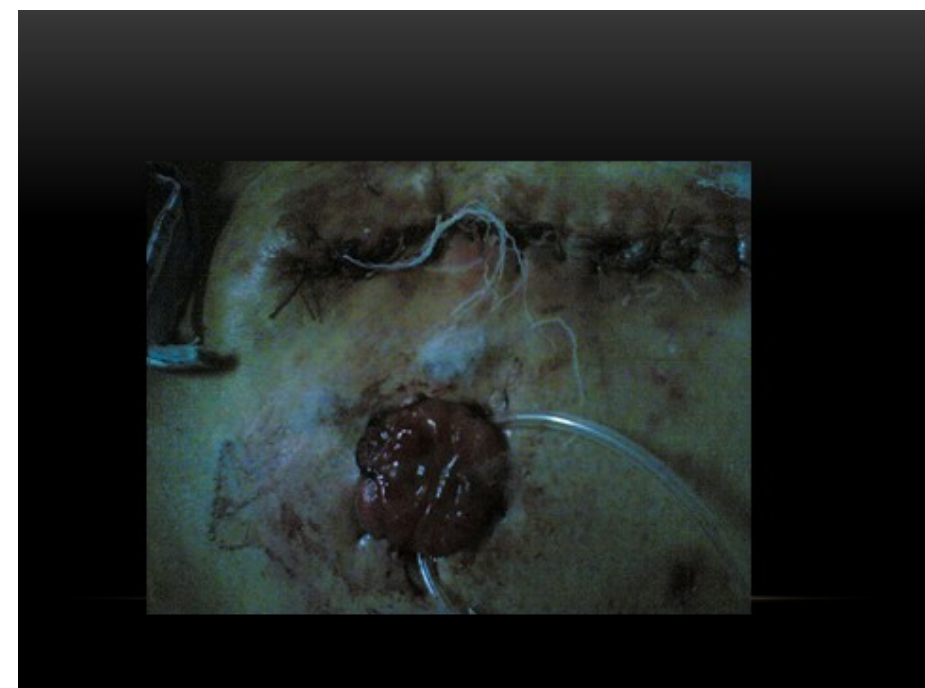

Fig 2..Fistula Repaired And Proximal Loop Ileostomy Done...Case-1

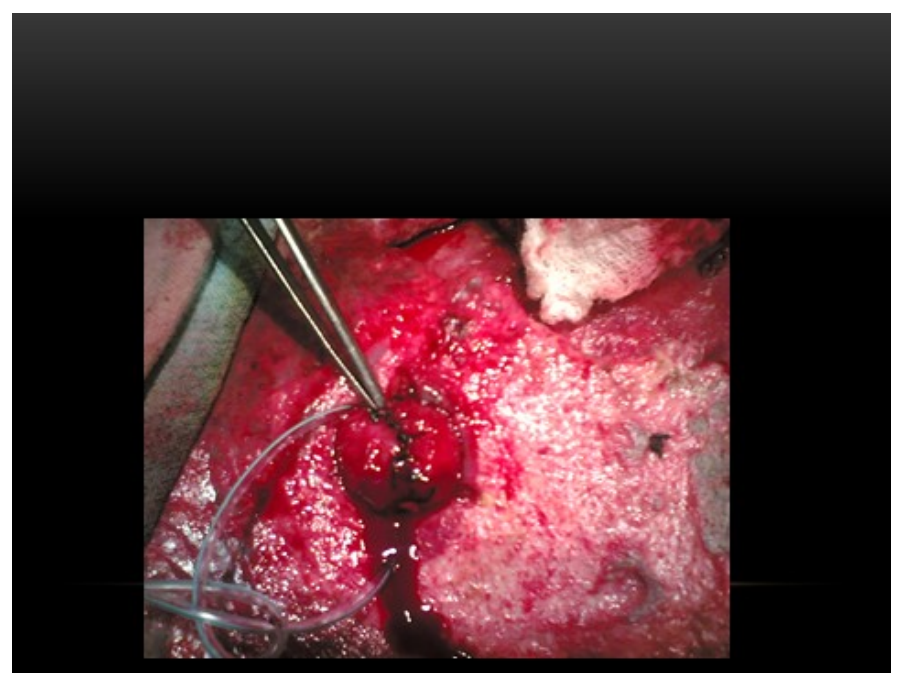

Fig 3..Ileostomy Closed And Loop Kept Outside To See For Leakge..Case-1

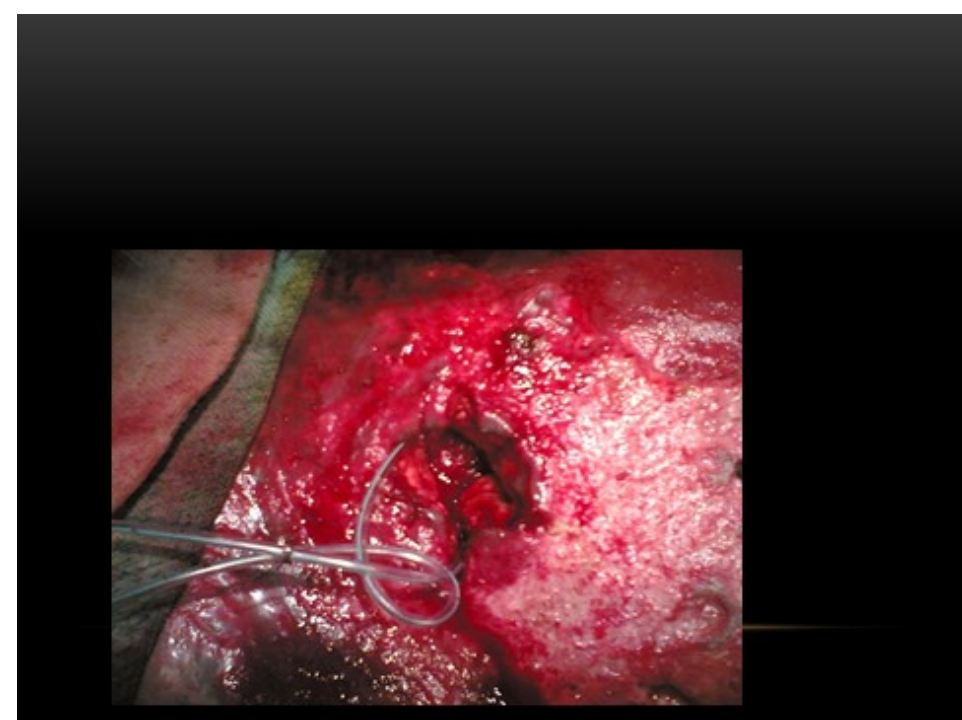

Fig 4. .Closed Ileostomy Put Inside Peritoneal Cavity But With Catheter Support . Case-1 


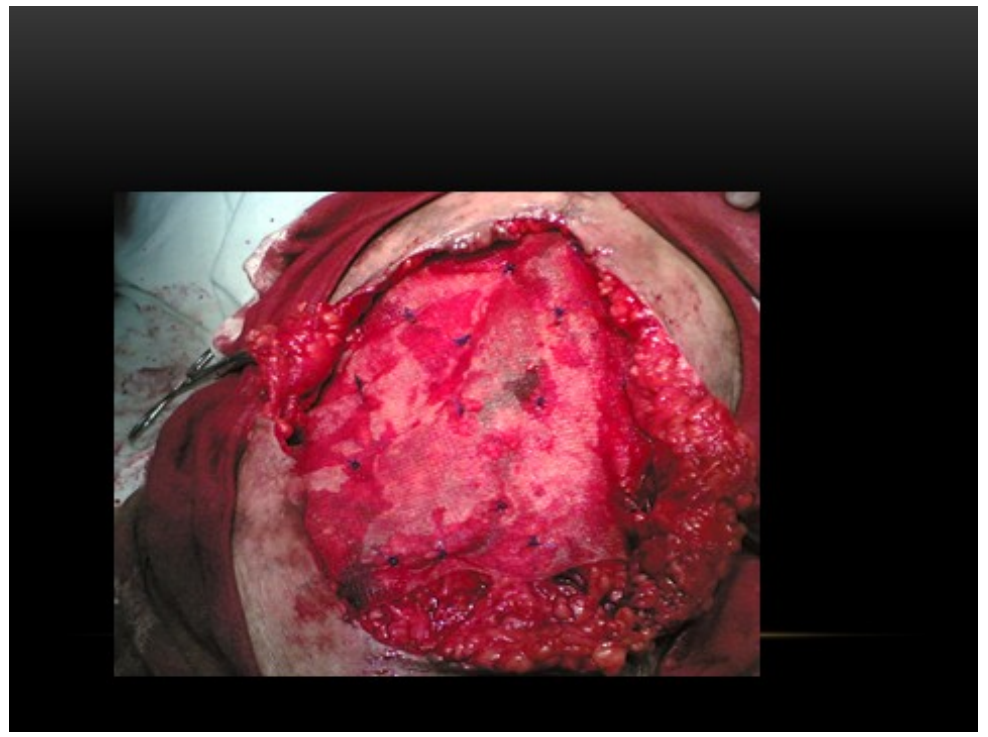

Fig 5..Patient Had Burst Abdomen.Abdominal Wound Closed With Mesh Interposition And To Wait For Healing By Secondary Intention. Case-1

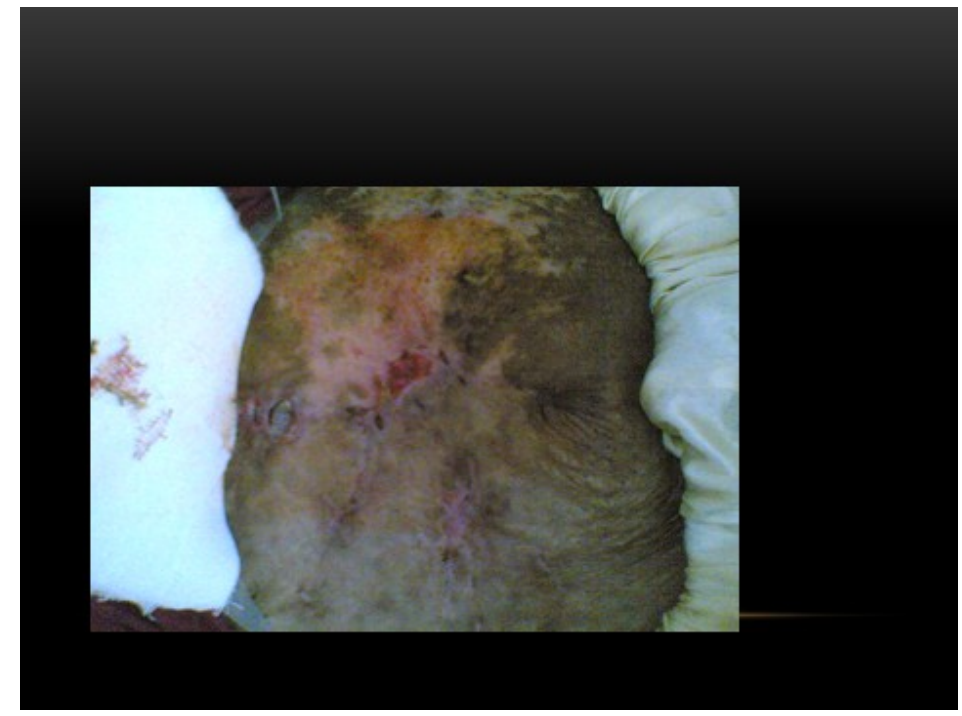

Fig 6..Completely Healed Wound .Case-1

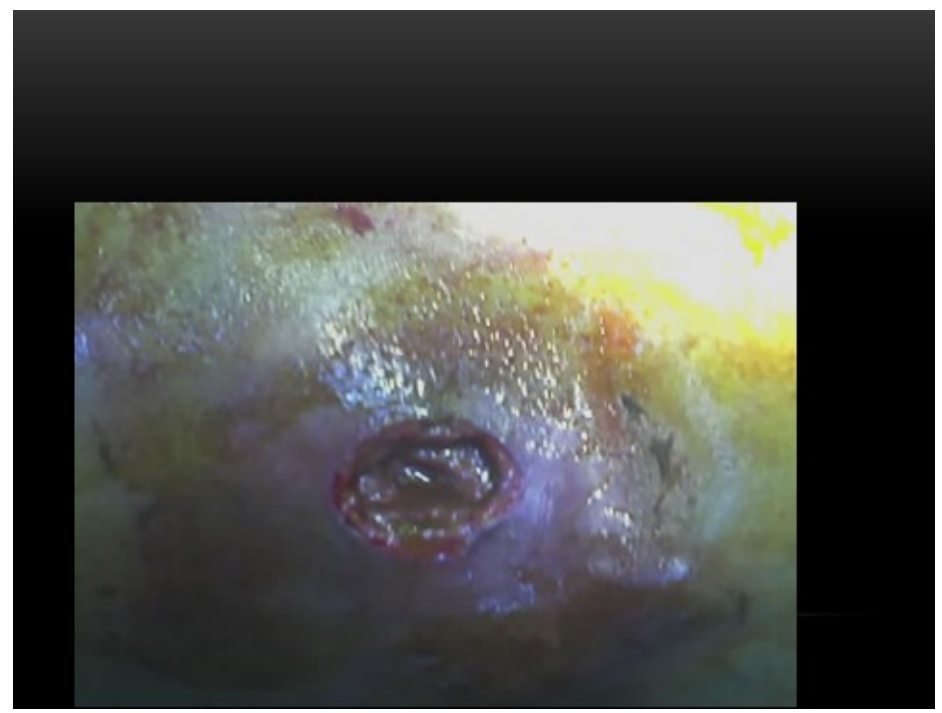

Fig 7..Enterocutaneous Fistula Case-2 


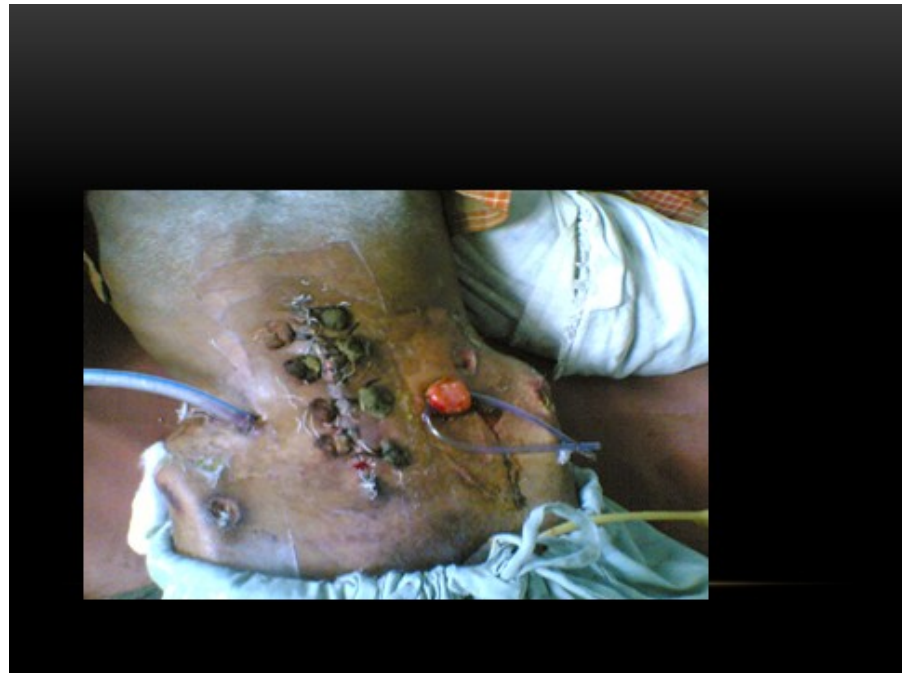

Fig 8..Fistula Repaired With Proximal Loop Ileostomy Case-2

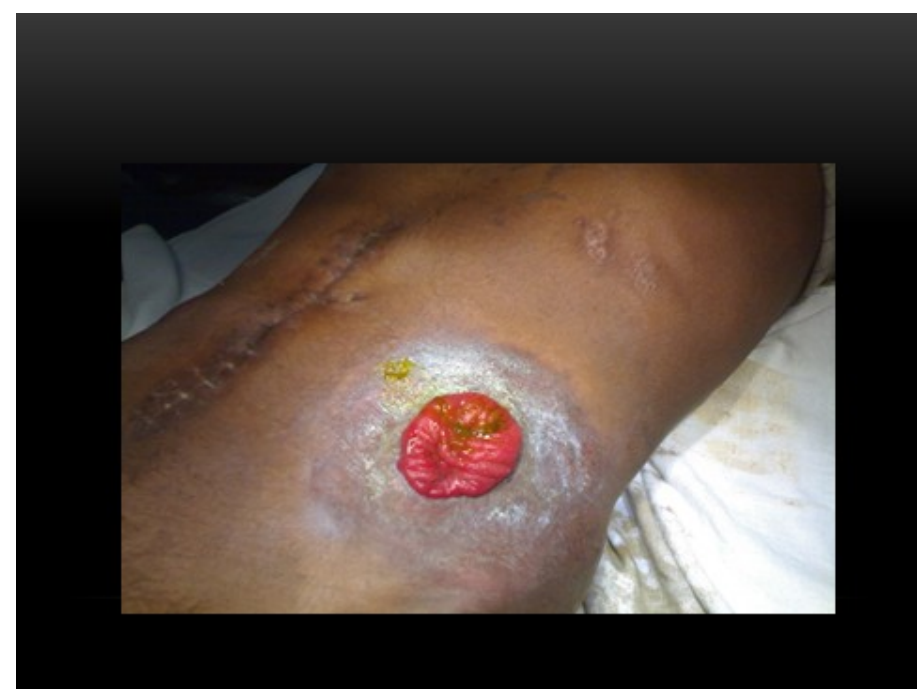

Fig 9..Wound Healed.Ileostomy Working. Case-2

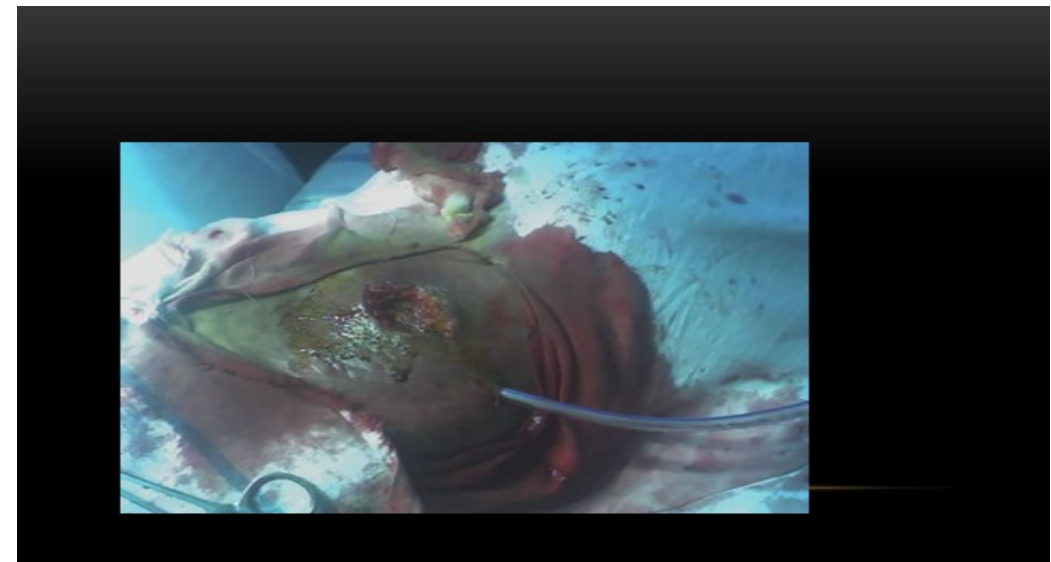

Fig 10..Enterocutaneous Fistula With Drain In Peritoneal Cavity. Case-3 


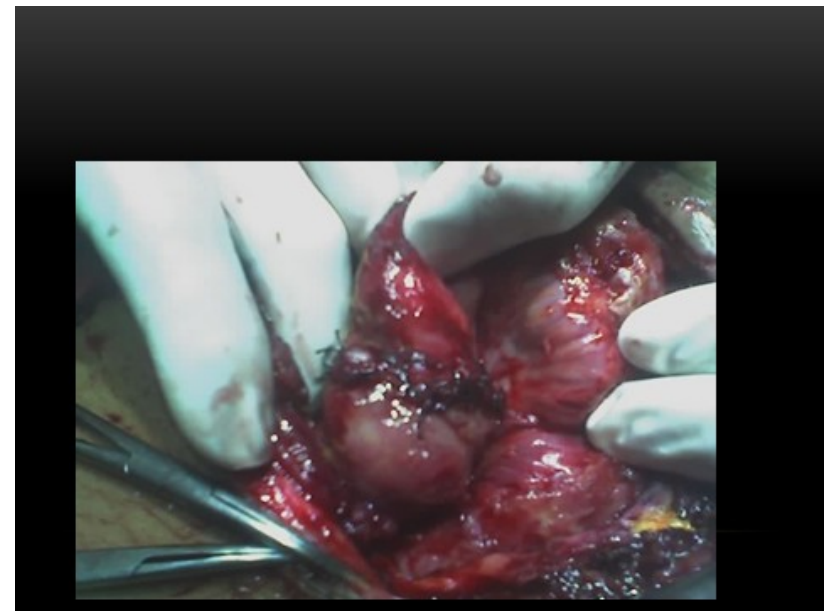

Fig 11.. Fistular Site Repaired .Case-3

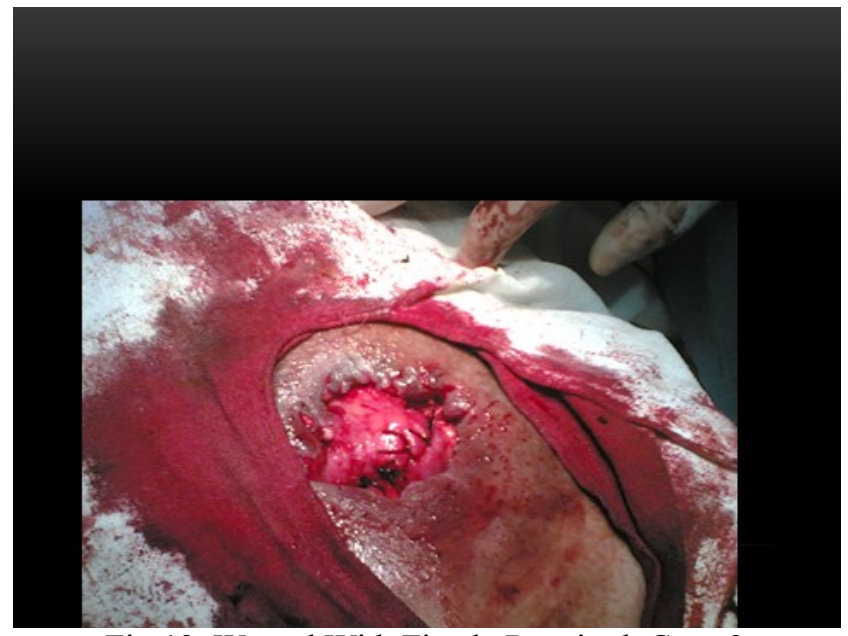

Fig 12..Wound With Fistula Repaired. Case-3

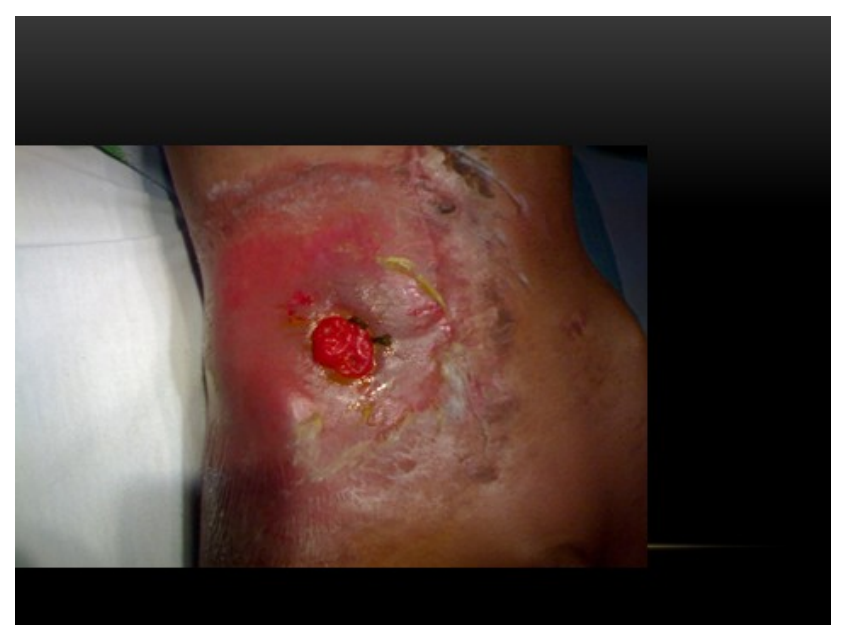

Fig 13..Healed Abdominal Wound With Ileostomy. Case-3 


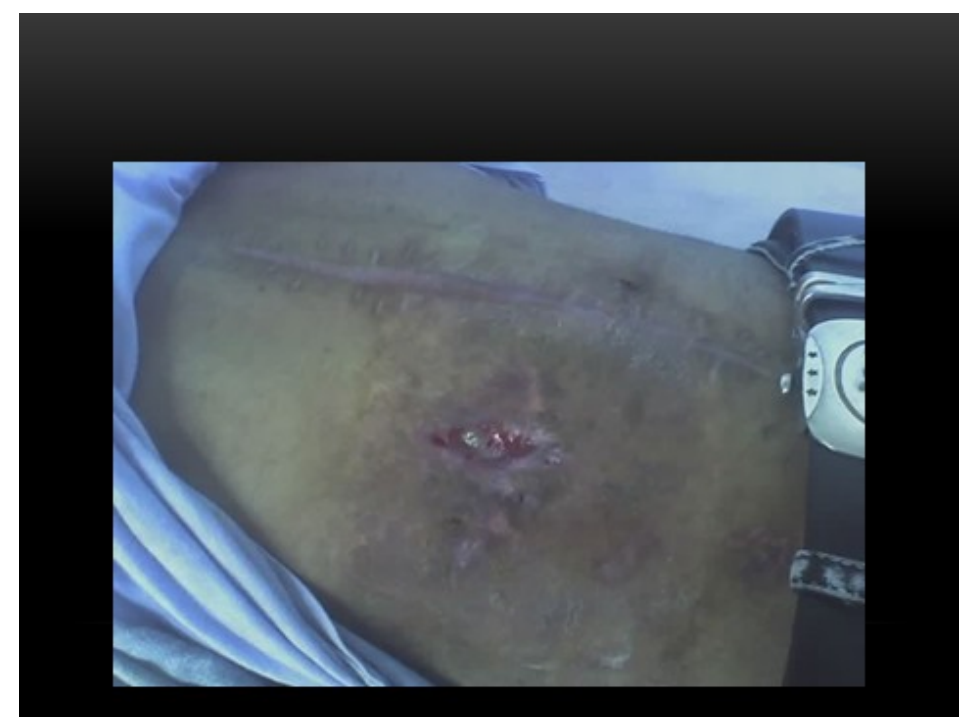

Fig 14..Completely Healed Wound After Ileostomy Closure. Case-3

\section{Conclusion}

- Our study concludes that in cases of complicated entero-cutaneous fistulas, early proximal loop ostomies/ileostomies along with the repair of fistular sites gives good results and less morbidity and mortality.

- This procedure can be considered in the management of complicated entero-cutaneous fistula.

- Medical and nursing care demand a complementary,interdisciplinary approach if successful closure of anenterocutaneous fistula is to be achieved. The patientandfamily are challenged by physical and psychologicalstressors, which often result in weeks and even months of hospitalization. As health-care practitioners, we must remember to treat the patient as a whole person and not just 'as a hole.' The fistula should notbecome the only focus of care, but rather an elementof the overall treatment plan.

\section{References}

[1]. Kumar P, Maroju NK, Kate V. Enterocutaneous fistulae: etiology, treatment, and outcome - a study from South India. Saudi J Gastroenterol. Nov-Dec 2011;17(6):391-5.

[2]. Berry SM, Fischer JE. Classification and pathophysiology of enterocutaneous3.fistulas. SurgClin North Am. Oct 1996;76(5):1009-18.

[3]. Edmunds LH Jr, Williams GH, Welch CE. External fistulas arising from the gastro-intestinal tract. Ann Surg. Sep 1960;152:445-71.

[4]. Fischer PE, Fabian TC, Magnotti LJ, et al. A ten-year review of enterocutaneous fistulas after laparotomy for trauma. J Trauma. Nov 2009;67(5):924-8.

[5]. Falconi M, Sartori N, Caldiron E, et al. Management of digestive tract fistulas. A review. Digestion. 1999;60Suppl 3:518.

[6]. Evenson AR, Fischer JE. Current management of enterocutaneous fistula. J Gastrointest Surg. Mar 2006;10(3):455-64.

[7]. Tong CY, Lim LL, Brody RA. High output enterocutaneous fistula: a literature review and a case study. Asia Pac J ClinNutr. 2012;21(3):464-9.

[8]. Campos AC, Andrade DF, Campos GM, et al. A multivariate model to determine prognostic factors in gastrointestinal fistulas. J Am Coll Surg. May 1999;188(5):483-90.

[9]. Reber HA, Roberts C, Way LW, et al. Management of external gastrointestinal fistulas. Ann Surg. Oct 1978;188(4):4607.

[10]. Draus JM Jr, Huss SA, Harty NJ, et al. Enterocutaneous fistula: are treatments improving?. Surgery. Oct 2006;140(4):570-6; discussion 576-8. 